\title{
OMAE2005-67460
}

\section{MODELING TWO-PHASE FLOW WITH OFFSHORE APPLICATIONS}

\author{
Rik Wemmenhove \\ Department of Mathematics \\ University of Groningen \\ PO Box 800 \\ 9700 AV Groningen \\ The Netherlands
}

\author{
Roel Luppes \\ Department of Mathematics \\ University of Groningen \\ PO Box 800 \\ 9700 AV Groningen \\ The Netherlands
}

\author{
Erwin Loots \\ Department of Mathematics \\ University of Groningen \\ PO Box 800 \\ 9700 AV Groningen \\ The Netherlands
}

\author{
Arthur E. P. Veldman \\ Department of Mathematics \\ University of Groningen \\ PO Box 800 \\ 9700 AV Groningen \\ The Netherlands
}

\begin{abstract}
With the trend towards offshore LNG production and offloading, sloshing of LNG in partially filled tanks has become an important research subject for the offshore industry. LNG sloshing may induce impact pressures on the containment system and may affect the motions of the LNG carrier.

So far, LNG sloshing has been studied mainly using model experiments with an oscillation tank. However, the development of Navier-Stokes solvers with a detailed handling of the free surface allows the numerical simulation of sloshing. It should be investigated, however, how accurate the results of this type of simulations are for this complex flow problem.
\end{abstract}

The paper first presents the details of the numerical model, an improved Volume Of Fluid (iVOF) method. The program has been developed initially to study the sloshing of liquid fuel in satellites. Later, the numerical model has been used for calculations of green water loading and the analysis of antiroll tanks, including the coupling with ship motions.

Recently, the model has been extended to incorporate twophase flow. This extension improves its ability to simulate the effect of gas bubbles of different sizes. Gas bubbles are present in virtually all relevant offshore situations; not only at
LNG sloshing but also during green water events, bow slamming and water entry.

In a two-phase flow model, both the liquid and the gas phase can have their own continuity and momentum equations. The handling of the compressibility of the gas phase is a major issue in the design of a two-phase flow model. However, as a first step in the modeling process, the gas phase is considered as incompressible.

For a dambreak experiment, results of the one-phase model, the incompressible two-phase model and model experiment results have been compared. It is shown that the physics are more accurately simulated with the incompressible two-phase model. Furthermore, the paper will show results of the incompressible model for LNG sloshing.

The physics of LNG sloshing and several other applications can be approached better by taking the compressibility into account. Therefore, as a second step, a compressible model is currently under construction, involving adiabatic compression of the gas phase.

\section{INTRODUCTION}

The simulations of many offshore flow problems are currently facing the physics of complex mixtures of fluids. Different combinations of fluids are of practical interest, for example 
the combination of water and air or the combination of LNG and air. The mixing of water and air affects the pressure and water levels on offshore structures for more or less severe hydrodynamic conditions. Typical situations where two-phase flow should be taken into account are bow slamming, water entry and green water loading.

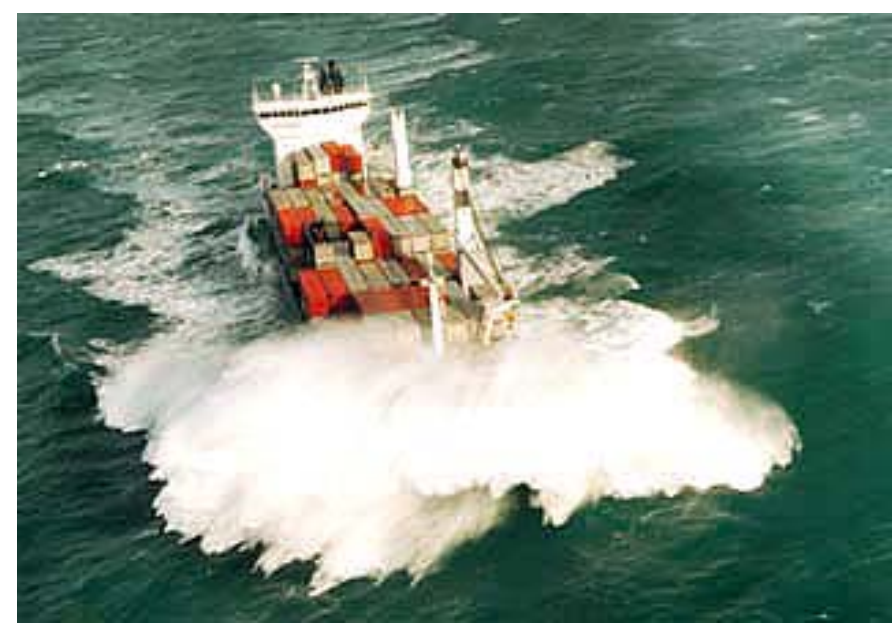

Figure 1: Example of green water loading

Sloshing of LNG and other media in tanks is of increasing interest, since ships are no longer sailing with full or empty tanks only. The current trend towards offshore LNG production and offshore discharge from LNG carriers may impose a challenge with respect to sloshing induced loads on the tank, as such operation involves intermediate filling of the cargo tanks when at sea.

So far sloshing loads have mainly been determined through model experiments. Model testing is expensive and time consuming and not practical in a design loop (e.g. for tank shape optimization aiming for sloshing load reduction). Therefore, a numerical tool that allows simulation of liquid motion inside slack LNG tanks and calculation of pressure loading on the tank boundaries is of great value, both as a design tool for relative evaluation of two designs and as a tool for predicting absolute values of pressure loading on the LNG tank containment system.

In the present paper a two-phase numerical model for simulation of sloshing and other offshore problems is presented together with two examples of application: the calculation of wave impact on a block structure (a dambreak simulation) and the calculation of sloshing induced loads on a membrane type LNG cargo tank. The results of the calculations are compared with experimentally obtained loads and with the results of a one-phase numerical model [7].

The first section of the paper describes the physical and mathematical framework of the numerical model. Compared with a one-phase numerical model, the implementation of a two-phase model requires the registration of more flow variables. After the physical-mathematical model, the details of the numerical model, i.e. an improved Volume OF Fluid (iVOF) method are presented. Thereafter, results of a dambreak simulation and of LNG sloshing simulations will be presented and discussed. Comparisons will be made with model test results for both cases. Aspects like water height evolution and impact pressure accuracy are discussed in the paper.

\section{PHYISCAL-MATHEMATICAL MODEL}

\section{Mathematical model for one-phase}

Taking only one-phase into account implies the use of the incompressible Navier-Stokes equations. These describe conservation of mass (Eq. 1) and momentum (Eq. 2):

$$
\begin{aligned}
& \nabla \cdot \vec{u}=0 \\
& \frac{\partial \vec{u}}{\partial t}+\nabla(\vec{u} \cdot \vec{u})+\frac{1}{\rho} \nabla p=\nabla^{2} \vec{u}+\vec{F}
\end{aligned}
$$

with $\vec{F}$ an external body force (such as gravity), pressure $p$, time $t$, a constant fluid density $\rho$ and a constant kinematic viscosity $v$.

\section{Addition of the second phase}

Addition of the second phase results in a nonuniform density distribution (see Fig. 2), so the continuity and momentum equations have to include density variations as well:

$$
\frac{\partial \rho}{\partial t}+\nabla \cdot(\rho \vec{u})=0
$$
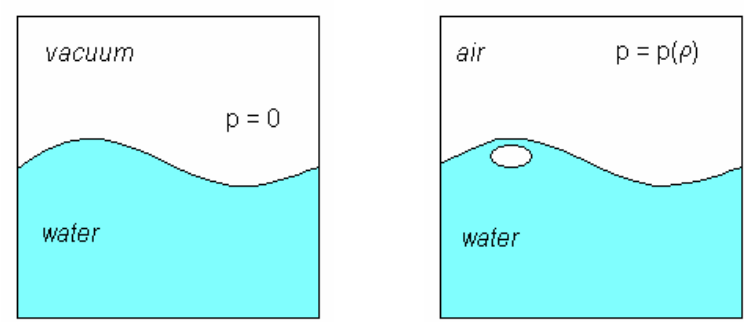

Figure 2 One-phase model (left) and two-phase model (right)

$$
\frac{\partial(\rho \vec{u})}{\partial t}+\nabla \cdot(\rho \vec{u} \vec{u})+\nabla p=\nabla \cdot(\mu \nabla \vec{u})+\rho \vec{F}
$$

In the momentum Eq. (2) of the one-phase model, all terms were divided by the constant density $\rho$; this is in contrast with the two-phase momentum Eq. (4). The local density can be calculated from the densities of the two fluids by weighted averaging. When the volume fraction of fluid 1 is given by $F_{1}$, the weighted density is given by

$$
\rho_{w g h t}=F_{1} \rho_{1}+\left(1-F_{1}\right) \rho_{2} \text {, }
$$

The local viscosity, which is constant in the one-phase model, is calculated as a harmonic average: 
$\mu_{\text {har }}=\left(\frac{F_{1}}{\mu_{1}}+\frac{1-F_{1}}{\mu_{2}}\right)^{-1}$

Assuming incompressibility of both phases reduces Eq. (3) and Eq. (4) to

$$
\begin{aligned}
& \nabla \cdot \vec{u}=0 \\
& \rho \frac{\partial \vec{u}}{\partial t}+\rho \nabla \cdot(\vec{u} \vec{u})+\nabla p=\nabla \cdot(\mu \nabla \vec{u})+\rho \vec{F}
\end{aligned}
$$

\section{STRUCTURE OF THE NUMERICAL MODEL}

The Volume Of Fluid (VOF) algorithm as developed by Hirt and Nichols [4] is used as a basis for the fluid advection. An overview of VOF and alternative methods available for the treatment of free surfaces and interfaces is given in [8].

For a one-phase approach, the current method solves the incompressible Navier-Stokes equations with a free-surface condition on the free boundary. In the VOF method a VOF function $F_{s}$ (with values between 0 and 1) is used, indicating the fraction of the cell that is filled with fluid. The free surface is reconstructed in each computational cell.

This is in contrast with a two-phase approach, when the air water interface is no longer considered as a free-surface, although the interface is still reconstructed using the VOF algorithm. The function $F_{s}$ is used to determine the aggregated density value inside a grid cell, using weighted averaging (Eq. 5). Next, the pressure value $p$ is calculated using the continuity and momentum equations.

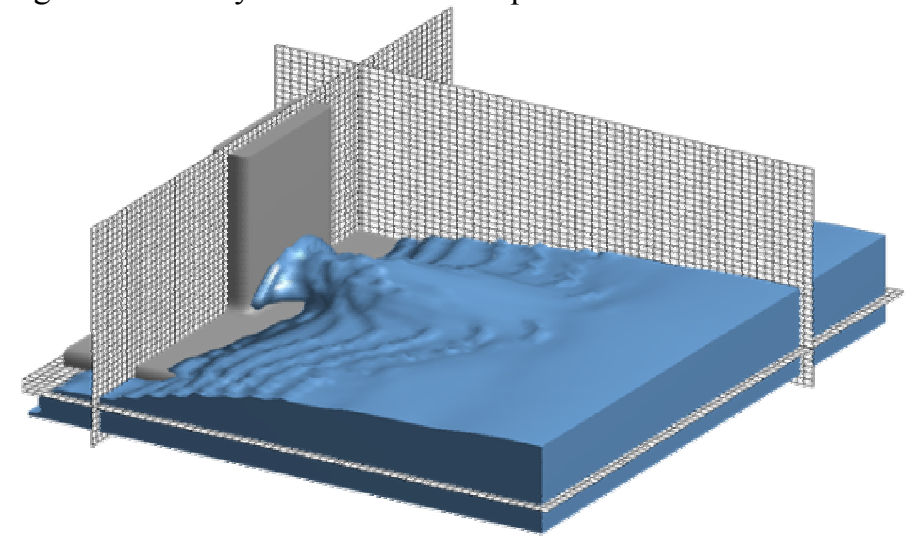

Figure 3: Wave impact simulation; the 3D grid is also shown.

ComFLOW is an improved 3D Volume Of Fluid (iVOF) Navier-Stokes solver. Compared with the original VOF algorithm, a local height function improves the treatment of the free surface. The program has been developed initially by the University of Groningen/RuG to study the sloshing of liquid fuel in satellites. This micro-gravity environment requires a very accurate and robust description of the free surface. In close co-operation with MARIN, this methodology was later extended to the calculation of green water loading on a fixed bow deck [3]. Also anti-roll tanks, including the coupling with ship motions [1], were investigated. Furthermore, the entry of a wedge in a fluid was studied as part of the RuG-MARIN co-operation [5], as well as the wave impact loads on fixed structures (see Figure. 3).

\section{Discretisation: incompressible flow model}

The discretisation of the Navier-Stokes equations is done on a staggered grid, which means that the pressure is set in the cell centres and the velocity components in the middle of the cell faces between two cells.

For incompressible conditions, the Navier-Stokes equations, as given by Eqns. (7) and (8), are discretised in time according to the explicit first order Forward Euler method:

$$
\begin{aligned}
& \nabla \cdot u^{n+1}=0 \\
& \rho^{n}\left(\frac{u^{n+1}-u^{n}}{d t}\right)+\rho^{n} \nabla \cdot(u u)^{n}+\nabla p^{n+1}= \\
& \nabla \cdot\left(\mu^{n} \nabla u^{n}\right)+\rho^{n} F^{n} \\
& \nabla\left(\frac{1}{\rho^{n}} \nabla p^{n+1}\right)=\frac{1}{d t}\left(\nabla \cdot u^{n}\right)-\nabla^{2}(u u)^{n}+ \\
& \nabla^{2}\left(v^{n} \nabla u^{n}\right)+\nabla \cdot F^{n}
\end{aligned}
$$

$\Delta \mathrm{t}$ is the time step and $n+1$ and $n$ denote the new and old time level, respectively. The conservation of mass in Eq. (9) and the pressure in Eq. (10) are treated on the new time level $n+1$ to assure that the new velocity field is divergence-free (no gain or loss of fluid). The pressure expression in Eq. (11) is determined by taking the divergence of Eq. (10) and subtracting the time-derivative of the conservation of mass (Eq. (9)).

To distinguish between the different characters of grid cells, the cells are labeled. The variable $F_{b}$ describes the fraction of a grid cell open for fluid, while the variable $F_{s}$ describes the fraction of a grid cell filled by the liquid phase. The NavierStokes equations are discretised and solved in cells containing at least one of the fluids.

Every cell is given a label to distinguish between boundary, air and fluid. Two classes of labeling exist: geometry cell labels and fluid cell labels. The geometry labeling at each time step divides the cells into three classes:

F(low)-cells:

All cells with $F_{b} \geq 0$

B(oundary)-cells:

(e) $\mathbf{X}$ (ternal)-cells:

All cells adjacent to a $\mathbf{F}$-cell

All remaining cells

The free-surface cell labeling is a subdivision of the F-cells. The subdivision consists of 3 subclasses:
E(mpty) cells:
S(urface) cells:
$\mathbf{F}^{\prime}$ (luid)-cells: 
Fig. 4 shows an example of geometry cell labelling and freesurface cell labelling for a wedge entering a fluid in downward direction.
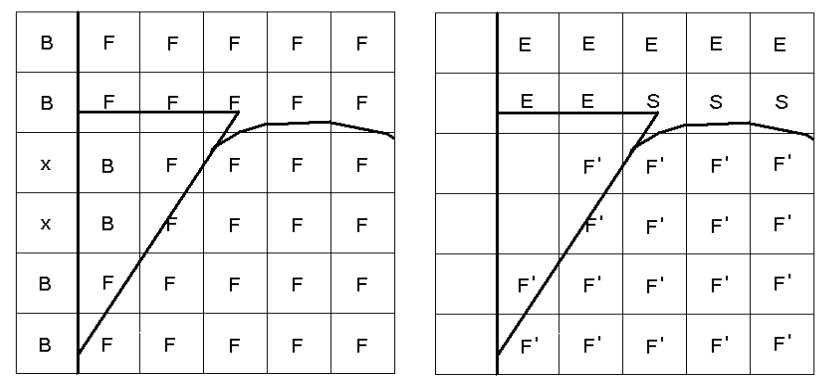

Figure 4: Geometry cell labeling (left) and free-surface cell label for a wedge entering a fluid (right)

The labeling system is retained in the two-phase model. The difference is that in the one-phase model the empty cells are truly empty in the sense that no computations are performed there, while boundary conditions for the free surface are prescribed around S-cells. In the two-phase model the empty cells denote the fluid with the smallest density; the NavierStokes equations are also solved in these cells.

The following functionalities which are relevant in the remainder of the paper are presently available in ComFLOW :

- Calculation of the fluid motion by solving the incompressible Navier-Stokes equations.

- Two (incompressible) fluids are considered

- Possibility to model an arbitrary number of fixed objects in the fluid.

- $\quad$ Options to use no-slip or free-slip boundary conditions at the solid boundaries. Inflow and outflow boundary conditions for fluid velocities and/or pressures can be defined.

- The fluid simulations are carried out on a Cartesian grid with user-defined stretching. The Cartesian grid is fixed in the domain. When the domain is moving (e.g. a LNG tank) a virtual body force is added to the forcing term in the Navier-Stokes equations. The fluid motions are thus solved in a domain-fixed co-ordinate system.

The next step in the development of the two-phase flow model is the extension to a compressible flow model. However, this extension is still under construction. This paper is limited to the comparison of the one-phase model with the incompressible two-phase model .

\section{MODEL RESULTS I : WAVE IMPACT AGAINST BLOCK STRUCTURE (DAMBREAK SIMULATION)}

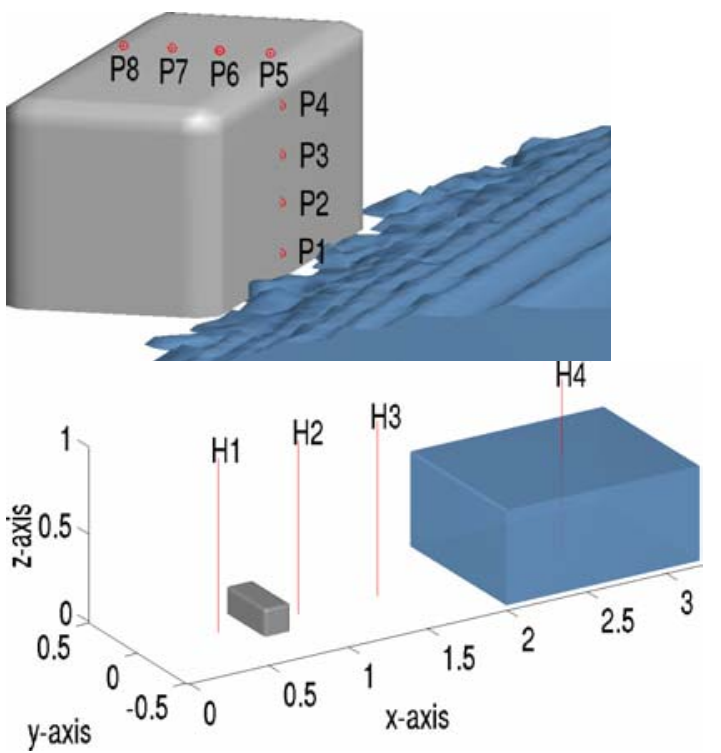

Figure 5: Measurement positions for pressure levels and water heights in the dambreak experiment. Pressure sensors are located on the small block.

At the Maritime Research Institute Netherlands (MARIN) experiments have been performed for breaking dam flows. These experiments can be seen as a simple model of green water flow on the deck of a ship.

The dambreak is a validation case characterized by an easy setup and can be used well to compare the results of the onephase flow model and the incompressible two-phase flow model.

The measurement setup consists of a large tank of 3.22 by 1 by 1 meter with an open roof [6]. The right part of the tank is first closed by a door. Behind the door 0.55 meter of water is waiting to flow into the tank when the door is opened. This is done by releasing a weight, which almost instantaneously pulls the door up. In the tank a box has been placed representing a scale model of a container on the deck of a ship. During the experiment measurements have been performed of water heights, pressures and forces. The small box is covered by eight pressure sensors, four on the front of the box and four on the top (see the top part of Fig. 5). Four vertical height probes are used, their positioning is shown in the bottom part of Fig. 5. The forces on the box are also measured. 


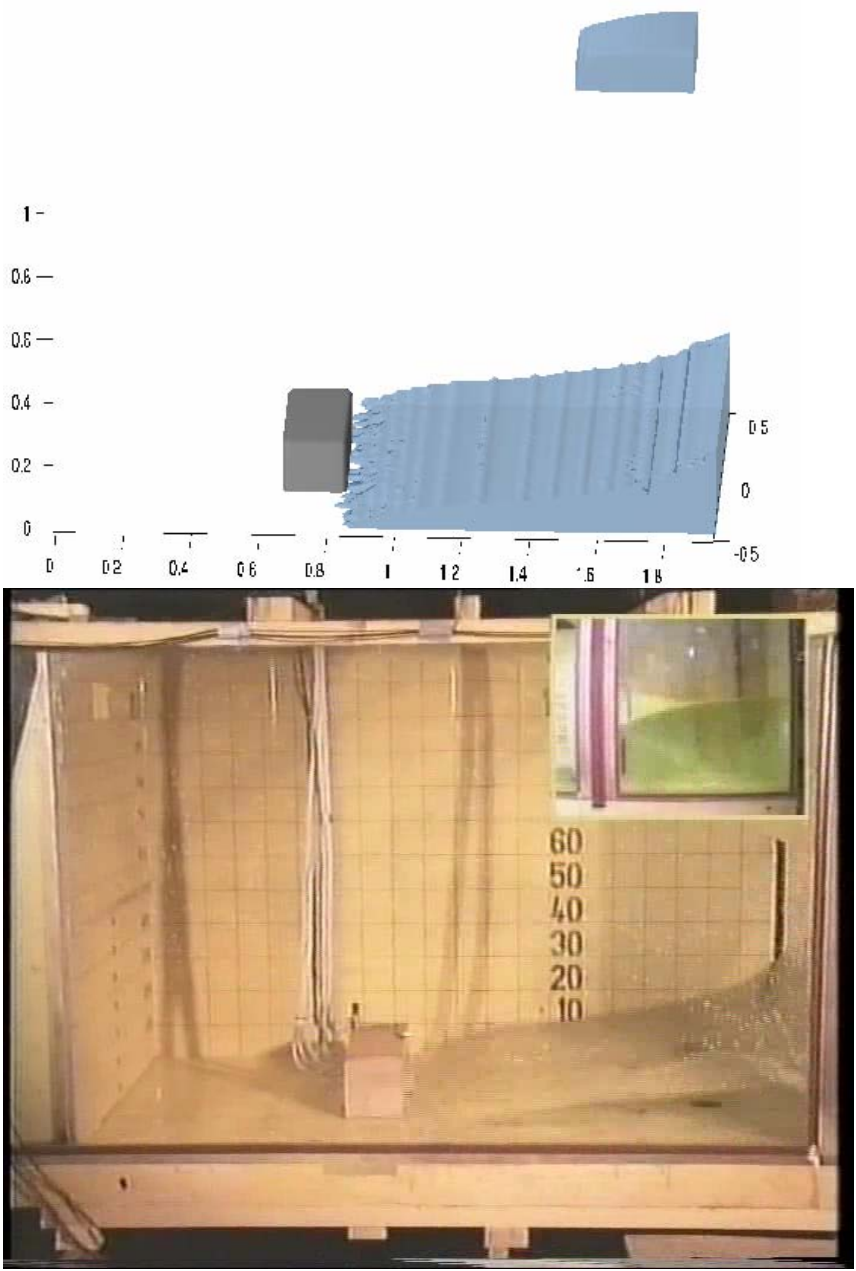

Figure 6a: Snapshot of a dambreak simulation with a box in the flow compared with experiment at time 0.4 seconds.

In the simulation with ComFLOW, the water in the right part of the domain is initially at rest, representing the initial condition where the door has just been removed. When the simulation is started the water starts to flow into the empty part of the tank due to gravity.

In Fig. $6 \mathrm{a}$ and Fig. $6 \mathrm{~b}$ two snapshots of the early stages of the simulation are shown together with some images of the video from the experiment (at the same instants in time).

Regarding Fig. 6a and Fig. 6b there is a good agreement between the snapshots of simulation and experiment. Although the one-phase flow simulation is able to represent the flow dynamics well, the two-phase flow simulation approaches the measurements even better. Fig. 7 shows the water height at position H1, just behind the block. The onephase model predicts a water level at $t=1 \mathrm{~s}$ that is clearly too high, and the rise time is too short. On the other hand, the results of the experiment and the two-phase model show a large resemblance, although the water in the two-phase simulation reaches station $\mathrm{H} 1$ a bit too late.

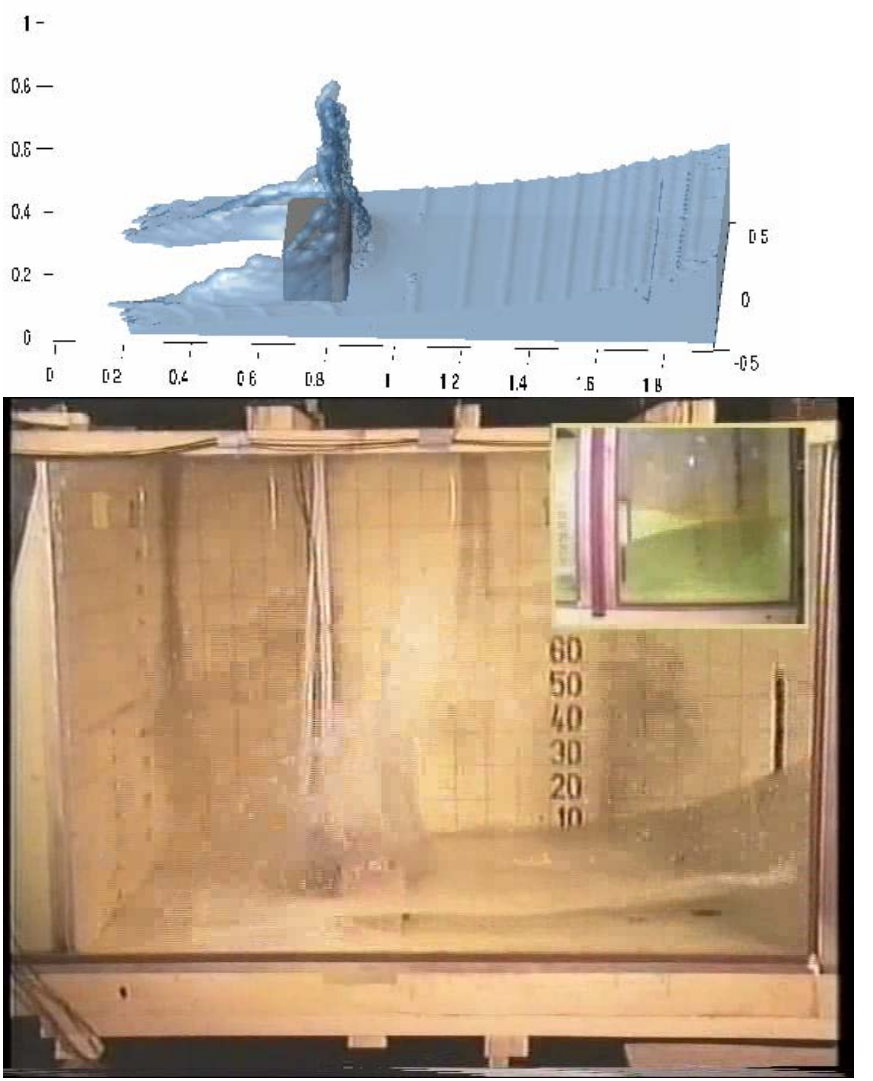

Figure 6b: Snapshot of a dambreak simulation with a box in the flow compared with experiment at time 0.56 seconds.

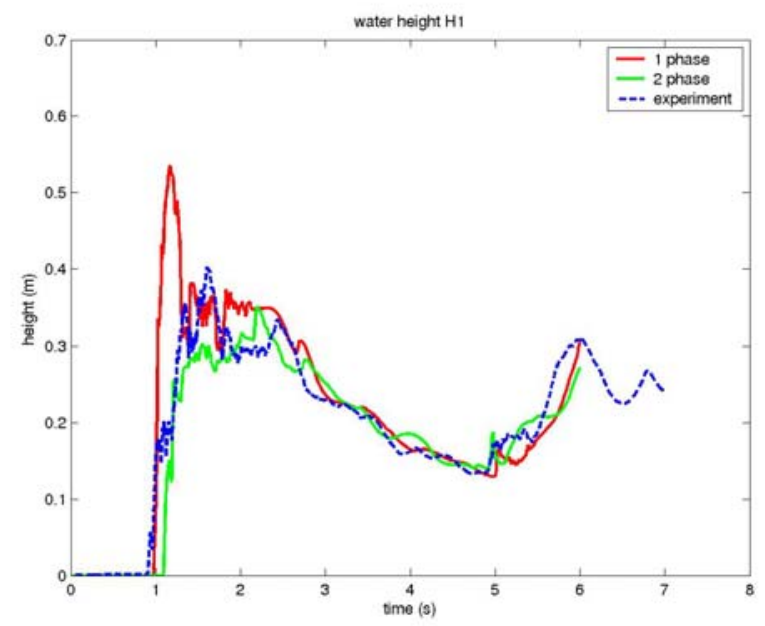

Figure 7 Water height at position $\mathrm{H1}$ (just left of the block); one-phase flow, two-phase flow and measurements.

The pressure level development on the front of the block is of practical interest as well, since these loads predict the stability 
of a container. Fig. 8 shows the pressure development at pressure sensor P3:

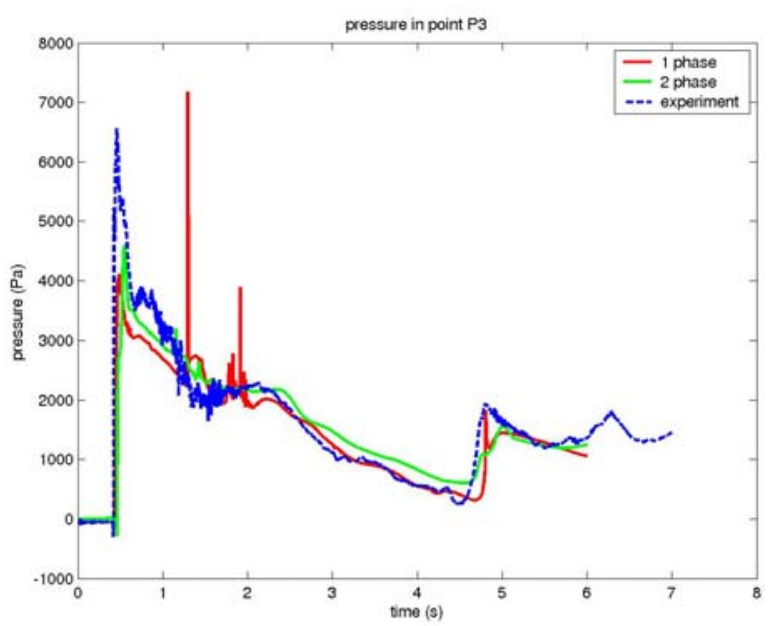

Figure 8: Pressure development at position P3 (at the front of the block); one-phase flow, two-phase flow and measurements.

For the pressure development the overall picture is quite similar to the water height. The initial pressure peak of the experiment is only estimated to a finite extent by the onephase simulation. The correlation between the two-phase simulation and the experimental results is better, while the one-phase simulation shows some artificial pressure spikes that are not present in the two-phase simulation. The origin of the artificial pressue spikes for the one-phase simulation will be further investigated for the LNG sloshing test case, see next section.

To summarize, the dambreak case shows that the experimental data is approached better by the two-phase simulation.

\section{MODEL RESULTS II : LNG SLOSHING IN A PARTIALLY FILLED TANK}

Another application of the two-phase flow model is sloshing in LNG tanks. For the purpose of obtaining experimental data to compare sloshing loads calculated by ComFLOW, a sloshing experiment with a $1 / 20$ scale model of a conventional size membrane type LNG carrier cargo tank was set up at Det Norske Veritas (DNV) in Norway (Figure 9). The tank model was excited with a regular sway motion at a frequency close to the natural period of the liquid for the particular filling height that was used. The filling was $70 \%$ of the tank height. The excitation period was 1.7 seconds and the amplitude of sway was 0.06 meters.

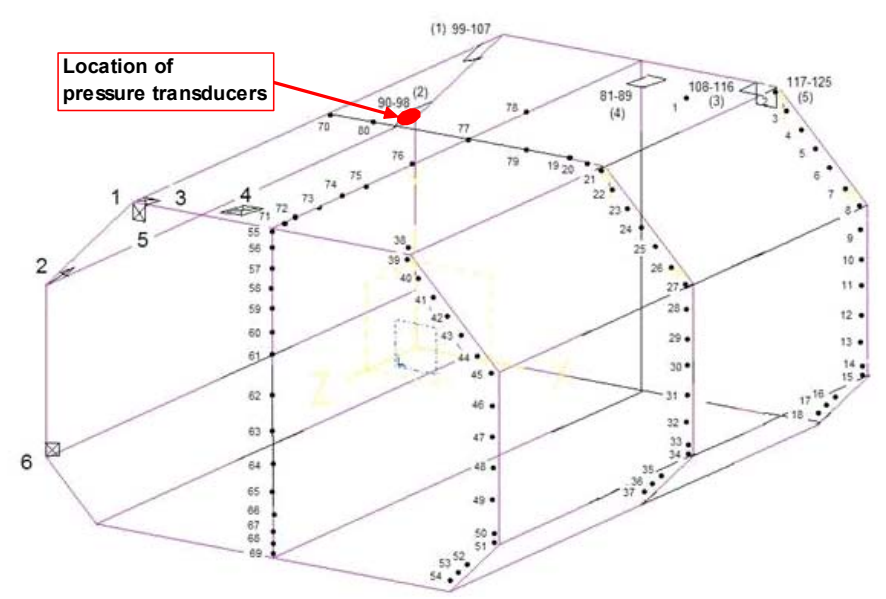

Figure 9: Schematic picture of the LNG tank model. Note the location of the pressure sensors in the corner.

The sloshing induced pressure loading on the lower knuckle of the upper chamber in way of the forward tank end was measured using a cluster of 9 pressure transducers within an area of 6 by $6 \mathrm{~cm}$. The sensor readings were averaged to represent the time history of pressure over 6 by $6 \mathrm{~cm}$. The location of the sensors is indicated in Fig. 9.

The sloshing experiment has been simulated by a one-phase version and by a two-phase version of ComFLOW. THe simulations have been performed in $2 \mathrm{D}$, the differences with a 3D simulation seemed to be insignificant. The calculations have been carried out for different grid sizes, with particular emphasis on pressure levels at the tank walls. The pressure level in the simulations has been evaluated at the same position as for the measurements (see Fig. 9).

\section{Observed flaws of the one-phase code}

Before looking at the results, we will briefly explain the relevant numerics that cause the differences.

In a typical sloshing simulation, the configuration of the free surface will often change quite rapidly. Topological changes will occur frequently. Therefore, it is very important to describe the velocities properly. Consider the situation in Fig. 10. The S-E velocity at the old time step changes to an F-S velocity at the new time step. In the one-phase model, the S-E velocity was set by using a boundary condition for the free surface. If this is not properly done, i.e. when the velocity field in the $\mathrm{S}$ cell is not divergence-free, the pressure has to 'work' to achieve mass conservation in the newly created F cell. This 'work' will manifest itself in a spike in the pressure signal.

Note that, especially in $3 \mathrm{D}$, many free surface configurations near solid boundaries occur which have to be taken care of. Especially when large amounts of fluid smash against a wall, as in the current application, pressure spikes cannot always be prevented. 


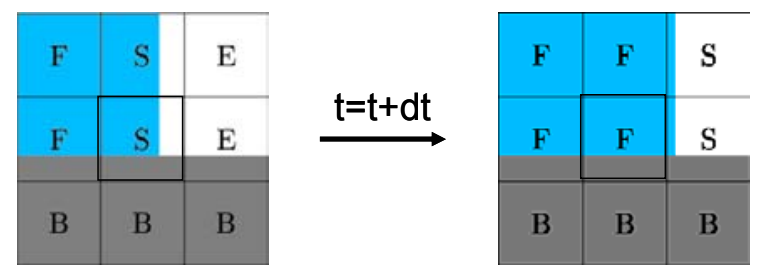

Figure 10: Motion of free surface changes the labeling.

Consider for example the situation in Fig. 11a and Fig. $11 \mathrm{~b}$.

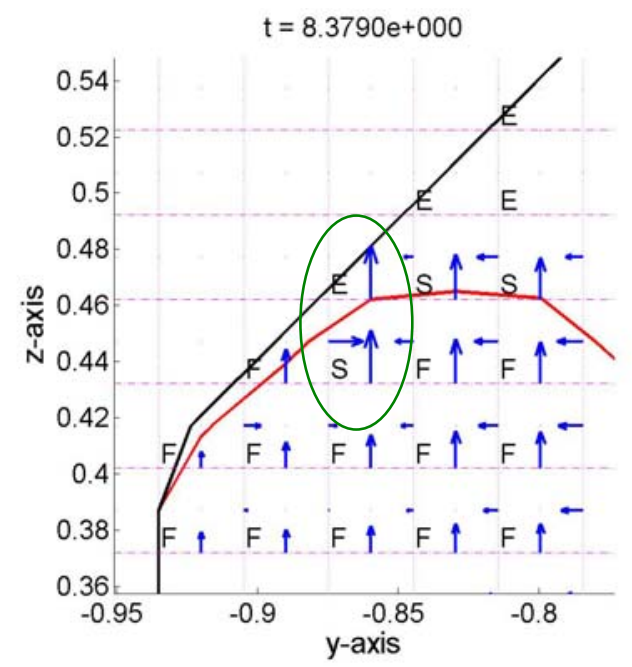

Figure 11a: Non-zero divergence outside flow leads to artificial high pressure peaks; previous timestep

The water front moves from the old time step (Fig. 11a) to the configuration shown in Fig. 11b. The (S,E) -pair of the circumlined cells becomes a pair of F-cells in a single time step. In E-cells and S-cells no conditions are satisfied with respect to mass conservation for the one-phase model. This can clearly be seen from the drawn staggered velocities at the edges of the two cells. Indeed, the vertical velocity between the S-cell and the E-cell in Fig. 11a is a boundary velocity which expresses continuity of velocity from the interior velocities outwards. However, in Fig. $11 \mathrm{~b}$ the cells have changed to F-cells. The sudden demand for divergence-free velocities in the newly created F-cells cannot be met without action by the pressure field: the pressure increases dramatically in order to adjust the velocity field. Note that the velocities drawn at the edges of the two F-cells now meet the divergence-free condition.

In order to solve this, E-F transitions are detected in the process. When such a transition has occurred, we look for a neighbouring boundary velocity and adjust this velocity such that mass conservation holds in the new F cell. Such a velocity can almost always be found when the free surface moves perpendicular to a wall. However, when slamming occurs, several labeling changes occur at once (as in this case) and no velocities to adjust are available. The pressure spike cannot be prevented and the height of the spike cannot be foreseen in these configurations. The actual spike belonging to the situation depicted in Fig. 11a and Fig. 11b can be seen in Fig. 13.

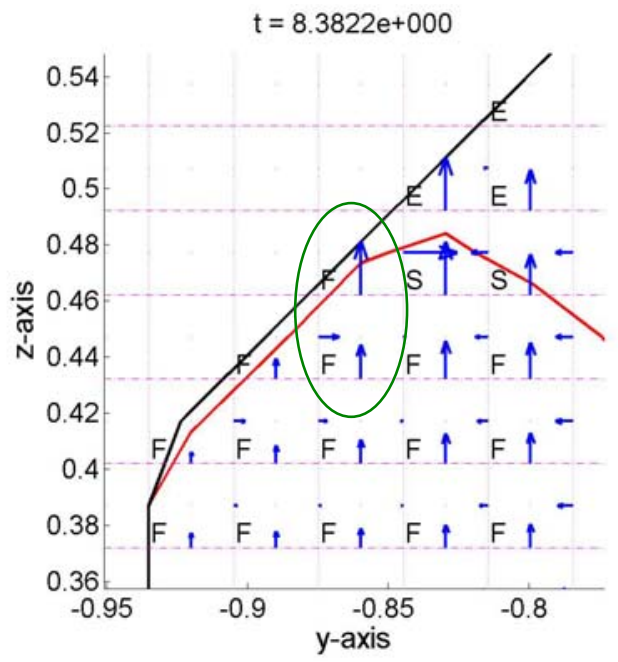

Figure 11b: Non-zero divergence outside flow leads to artificial high pressure peaks; next timestep

Here, the pressure signal becomes affected due to slamming, beacuse the velocity field is discontinuous. In the two-phase model, however, the continuity of the velocities over the interface ensures conservation of mass in both fluids (liquid and air). This will lead to a smoother pressure signal.

\section{Comparisons between one-phase model, two- phase model and measurements}

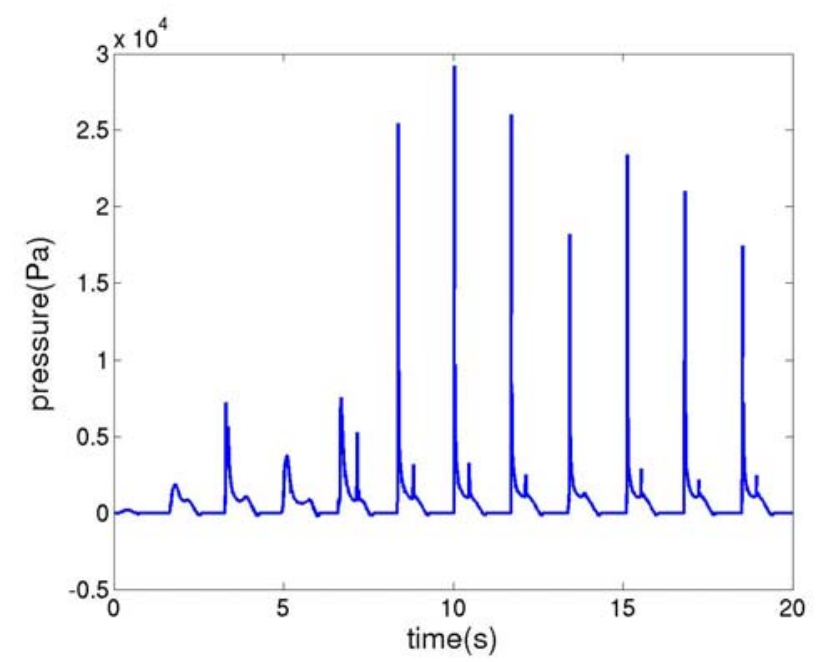

Figure 12: Time series of the pressure pulses: one-phase model

In Fig. 12, the time series of the pressure signal at the wall (y $=-0.91, \mathrm{z}=0.43$ ) is depicted for the one-phase code. Note the large variation in the pressure peaks. This variation is caused by the artificial pressure peaks as explained in the section above. Besides this, most pulses look rather similar. Fig. 13 is a typical example of an artificially high pressure peak. The circumlined fluctuations in the right part of Fig. 13 are the result of several label changes at once. 

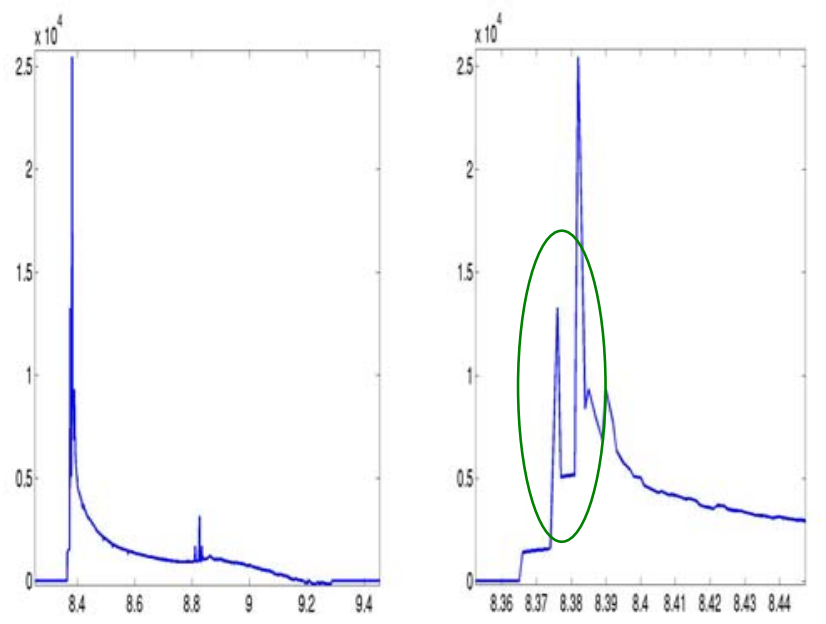

Figure 13: Pressure level development for the one-phase model at pos. $1(y=-0.91, z=0.43)$ in Fig. 9: $5^{\text {th }}$ excitation period. Left: entire pulse; right: first part of the pulse.

The two-phase code (Fig. 14) produces far less variation in peak pressures; moreover, the signal is much smoother with respect to the individual pulses. The flaws of the one-phase model, stemming from the discontinuous velocity field, are not present here. Indeed, the velocity field extends naturally into the airy part of the tank, as can be seen in Fig. 15.

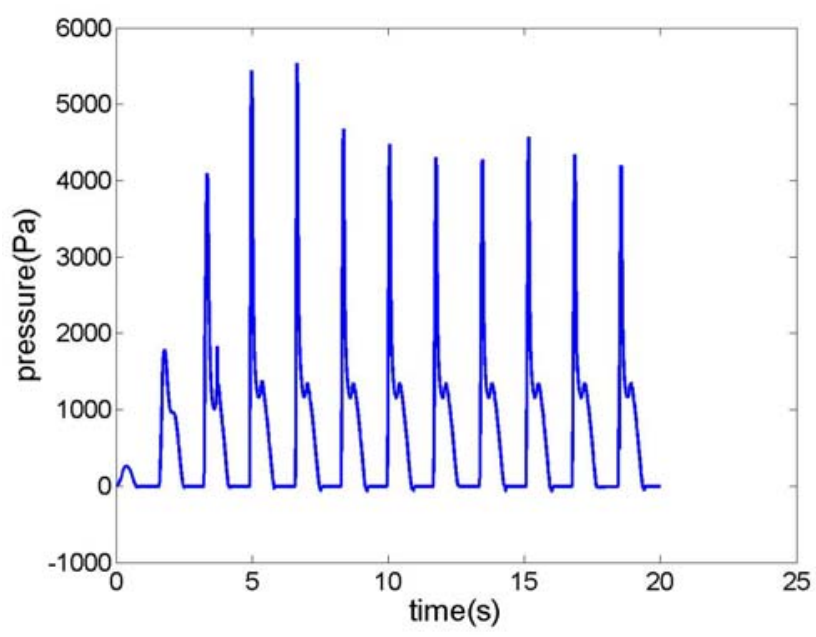

Figure 14: Time series of the pressure pulses: two-phase model

Comparing both pressure signals with the measurements from the experiment, it is found that the pressure pulse of the twophase model is much more regular than for the one-phase model. In Fig. 16 all pressure pulses of the experiment are drawn simultaneously, i.e. many excitation cycles are put together in a plot of one cycle. The pressure pulses of the onephase simulation and the two-phase simulation are drawn in the same way in Fig. 17. The simulations show more spreading than the experimental results. However, some of the peaks in the one-phase model seem exaggerated while the pulses in the two-phase code are more aligned.

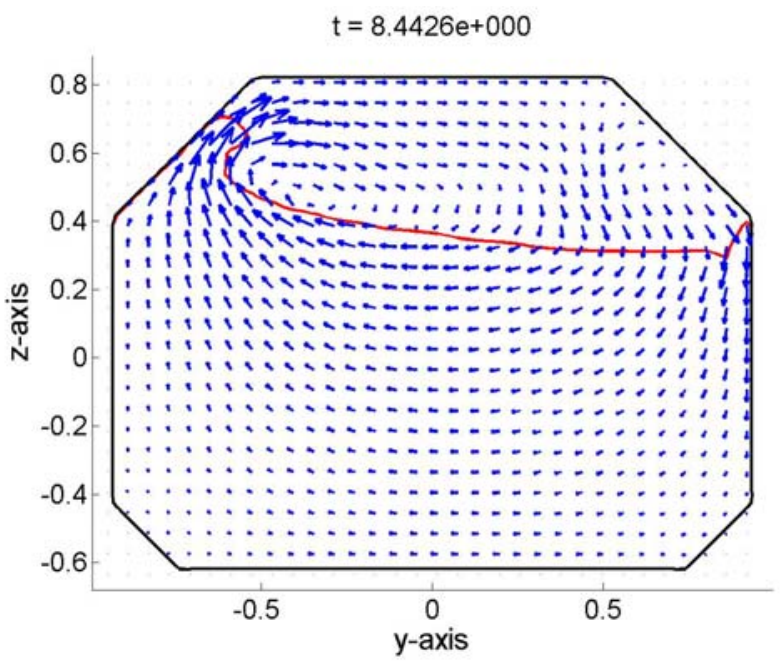

Figure 15: Snapshot of the velocity field in the two-phase simulation. The velocity field is continuous in both fluids and across their interface.

From the experiments it became clear that the pressure peak is rather local. Therefore, for better comparisons the simulations need to be carried out in 3D and on a finer grid. Moreover, the influence of compressible air pockets may play an important role and the modeling of air compressibility is therefore the next development step.

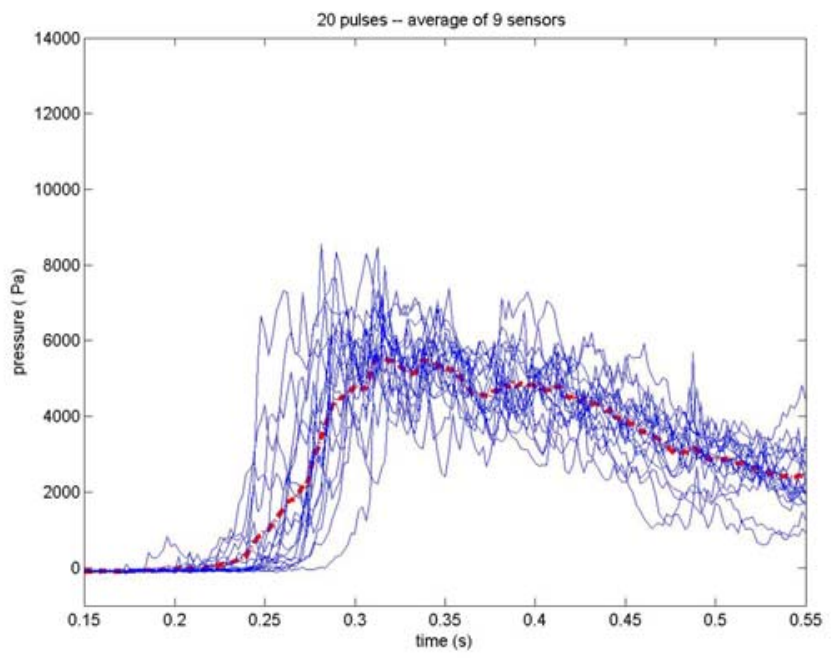

Figure 16: Series of pulses from the experiment 


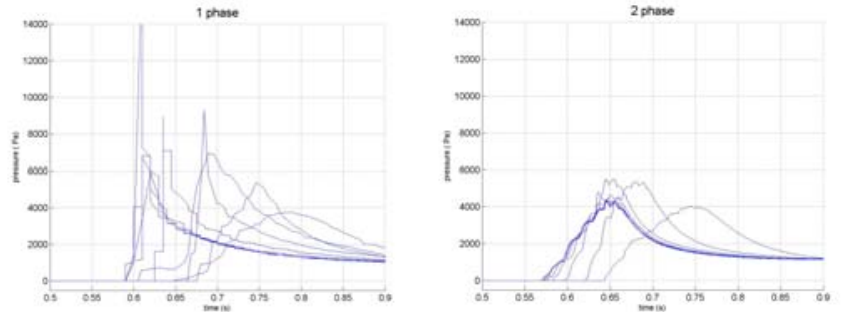

Figure 17: Series of pulses from the one-phase simulation (left) and the two-phase simulation (right) .

\section{CONCLUSIONS}

Based on the results presented in this paper, the following conclusions seem justified:

- This paper has shown that the hydrodynamics of different offshore applications can be simulated numerically using an iVOF Navier-Stokes solver.

- Using an incompressible two-phase numerical model approaches measurement results better than the onephase model.

- Compared with a one-phase flow model, the twophase flow model generates less (unphysical) pressure spikes, which enhances the simulation accuracy. The improvement stems from the fact that the entire velocity field is divergence-free.

- In order to attain more reliability, especially in the case of LNG sloshing simulations, compressibility of the air has to be taken into account.

\section{ACKNOWLEDGEMENTS}

This research is supported by the Dutch Technology Foundation STW, applied science division of NWO and the technology programme of the Ministry of Economic Affairs. The authors are grateful to MARIN for the data of the dambreak experiment and wish to thank DNV for making their data of the sloshing experiment available.

\section{REFERENCES}

1. Van Daalen, E. F. G., Kleefsman, K. M. T., Gerrits, J., Luth, H.R., Veldman, A.E.P., "Anti Roll Tank Simulations with a Volume of Fluid (VOF) based Navier-Stokes Solver", 23rd Symposium on Naval Hydrodynamics, Val de Reuil, 2000.

2. Gudmundsson, J.S., Cellius, H.K., "Gas-liquid metering using pressure-pulse technology”, SPE Annual Technical Conference and Exhibition, Houston, 1999.

3. Fekken, G., Veldman, A.E.P. and Buchner, B., "Simulation of Green Water Flow Using the NavierStokes Equations", Seventh International Conference on Numerical Ship Hydromechanics, Nantes, 1999.

4. Hirt, C.W. and Nichols, B.D., "Volume Of Fluid (VOF) Method for the Dynamics of Free Boundaries", Journal of Computational Physics, 39, pp 201-225, 1981.

5. Kleefsman, K.M.T., Fekken, G., Veldman, A.E.P., Bunnik, T., Buchner, B. and Iwanowski, B.; "Prediction of green water and wave impact loading using a Navier-Stokes Based Simulation Tool”, $21^{\text {st }}$ Offshore Mechanics and Arctic Engineering Conference, paper 28480, Oslo, 2002

6. Kleefsman, K.M.T., Fekken, G., Veldman, A.E.P., Buchner, B. and Iwanowski, B.; "A Volume-Of-Fluid Based Simulation Method For Wave Impact Problems", accepted for publication in Journal of Computational Physics, 2005.

7. Loots, G.E. and Buchner, B., "The numerical simulation of LNG sloshing with an improved Volume-of-Fluid method", $23^{\text {rd }}$ Offshore Mechanics and Arctic Engineering Conference, paper 51085, Vancouver, 2004.

8. Scardovelli, R. and Zaleski, S., "Direct Numerical Simulation of Free-Surface and Interfacial Flow", Annual Review of Fluid Mechanics, 31, pp 567-603, 1999.

$\begin{array}{ll}\text { NOMENCLATURE } \\ \mathrm{a} & {\left[\mathrm{ms}^{-1}\right]} \\ \mathrm{F} & {[\mathrm{N}]} \\ \mathrm{F}_{1} & {[-]} \\ \mathrm{F}_{\mathrm{b}} & {[-]} \\ \mathrm{t} & {[\mathrm{s}]} \\ \mathrm{u} & {\left[\mathrm{ms}^{-1}\right]} \\ \Delta \mathrm{t} & {\left[\mathrm{s}^{-1}\right.} \\ \mu_{1} & {\left[\mathrm{kgm}^{-1} \mathrm{~s}^{-1}\right]} \\ \mu_{2} & {\left[\mathrm{kgm}^{-1} \mathrm{~s}^{-1}\right]} \\ \mu_{\text {har }} & {\left[\mathrm{kgm}^{-1} \mathrm{~s}^{-1}\right]} \\ \nu & {\left[\mathrm{m}^{2} \mathrm{~s}^{-1}\right]} \\ \rho & {\left[\mathrm{kgm}^{-3}\right]} \\ \rho_{1} & {\left[\mathrm{kgm}^{-3}\right]} \\ \rho_{2} & {\left[\mathrm{kgm}^{-3}\right]} \\ \rho_{\mathrm{wght}} & {\left[\mathrm{kgm}^{-3}\right]} \\ \mathrm{n} & {[-]} \\ \mathrm{n}+1 & {[-]}\end{array}$

speed of sound

body force

volume fraction fluid 1

cell fraction open for flow

time

velocity

time step

dynamic viscosity fluid 1

dynamic viscosity fluid 2

harmonic viscosity

kinematic viscosity

density

density fluid 1

density fluid 2

weighted density

old time level

new time level 